\title{
Study on Corporate Social Responsibility Based on the Dissipative Structure Theory
}

\author{
Baoying Wang ${ }^{1,2}$ \\ ${ }^{1}$ School of Management and Economics,Beijing Institute of Technology,Beijing,100081,China \\ ${ }^{2}$ College of Economics and Management, North University of China,Taiyuan,030051, China \\ email: baoying_wang@163.com
}

Keywords: Corporate Social Responsibility; Dissipative Structure Theory; Mathematical Model of Dissipative Structure; Coordination Mechanism

\begin{abstract}
Fulfilling social responsibility is great significant for achieving sustainable development and building a harmonious society.Based on the Dissipative structure theory and from the system viewpoint,the internal and external conditions for the enterprise to form the dissipative structure are analysed.The mathematical models are developed to discuss the formation mechanism of dissipative structure in fulfilling social responsibility.Meanwhile,the coordination of the system is also analysed.
\end{abstract}

\section{Introduction}

We are puzzled by Chinese enterprises do not actively fulfill social responsibility even trample upon it.Repeatedly occurring affairs lose ethics,endanger the society and people health.The government,society and consumers' sanctions and supervisions are not the driving force of fulfillment of social responsibility. We have to rethink the reasons why the enterprises challenge the bottom line of ethics and harm the interests of the masses under the background of Chinese improving legal environment,close government supervision and public opinion tremendous force.

The dissipative structure is the important component of self-organization phenomenon,means under the condition of opening and far-from equilibrium,the system form persistent macro-ordered structure through the energy dissipation and internal non-linear dynamic mechanism in the process of material and energy exchange with outside[1].

The paper studies the fulfillment of social responsibility based on the Dissipative structure theory,analyses the internal conditions,external factors and how to form the dissipative structure of fulfilling social responsibility,and further discusses how to maintain the dissipative structure form the system viewpoint, points out the thoughts of fulfilling social responsibility.

\section{The Internal Conditions and External Factors of the Dissipative Structure Formation in Enterprise System}

Assuming the supplier and the manufacturer form the enterprise system.Two decision-makers are the supplier and the manufacturer,the demand of the manufacturer is random.The supplier provide a kind of spare part which is purchased by the manufacturer and used in the production.

According to the dissipative structure theory,the formation of dissipative structure is helpful to reach the order situation in time,space and function when the enterprise to fulfill social responsibility,avoid not fulfilling social responsibility or fulfilling disorderly.So,it is necessary to discuss the internal and external factors that influence on the formation of dissipative structure in enterprise system.

\section{The Internal Factors of the Dissipative Structure Formation}

Obviously,the enterprise system have clear characteristics of the dissipative structure formation.Firstly,the enterprise system is open.The enterprise system continuously exchanges materials,energies and informations with external environment,including social environment and natural environment.This is a interactive process.Secondly,the enterprise system is 
far-from-equilibrium.Through the exchange with external environment,the interior of system presents the characteristics of far from even and single.It is a active state which have certain regularity and good circulation state.Finally, The enterprise system is non linear.The relationship among the sub-systems within the enterprise system is not a simple cause-effect or superposition relationship,but a kind of complex corelationship.The sub-systems coordinate each other and formulate the new function which every sub-system does not possess[2, 3].

The External Factors of the Dissipative Structure Formation

The evolution of the enterprise system is also influenced by materials, energies and informations from external environment.Some external parameters provided by external environment are called external control parameter.They are the important external driving force of dissipative structure formation and influence the system development. Apparently,the formation,maintenance and development of the system need external driving force come from continuously exchange of materials,energies and informations.Moreover,the opening,non- equilibrium and non linear are the itself characteristics which form the dissipative structure.

\section{The Dissipative Structure Formation in Enterprise System When Fulfilling Social Responsibility}

The Analysis of the Mathematical model of the Dissipative Structure

Ilya Prigogine considered the far-from-equilibrium and opening system may form the dissipative structure and established a total entropy change model,that was the mathematical model of the dissipative structure.The model is shown as Equation (1).

$d s=d s_{i}+d s_{e}$

In (1), $d s$ is the system total entropy change in fulfilling social responsibility, $d s_{i}$ is the system positive entropy change in fulfilling social responsibility, that is internal increasing entropy,and $d s_{e}$ is the system negative entropy change in fulfilling social responsibility,that is external inputting negative entropy.The system would rely on the input and output of materials,informations and energies spontaneously and continuously to maintain its opening and far-from-equilibrium,keep its ordered structure[4, 5].

The fulfillment of social responsibility are related to the all respects in the enterprise's operation and management. The increase of $d s_{i}$ mainly come from the enterprise and its managers only pursuit the profit maximization,disregard the social responsibility ideas and practices.Thus,the enterprise choose to ignore responsibility and keep current situation. The increase of $d s_{e}$ mainly come from the input of advanced concepts and practices in the process of exchange materials,informations and energies with outside.Thus,the enterprise have more wider vision and the concept of sustainable development,discard the old thought and have more deep understanding in social responsibility[6, 7].

The Assumption and Further Analysis of the Mathematical Model

Assume that the materials,informations and energies from external and internal are $Q_{e}$ and $Q_{i}$ respectively,and define the exchange volume of materials,informations and energies per unit time as fluctuation,that is, $d Q_{e} / d_{t}$ and $d Q_{i} / d_{t}$ represent the external and internal fluctuation of the enterprise system.So the Equation (1) can be written as

$$
d s=d s_{i}+d s_{e}=-\frac{d Q_{e}}{d t}+\frac{d Q_{i}}{d t}
$$

When the value of time variable is larger, the Equation (2) can be expressed as

$$
\Delta s=\Delta s_{e}+\Delta s_{i}=\frac{\Delta Q_{e}}{\Delta t}+\frac{\Delta Q_{i}}{\Delta t}
$$

From Equation (3),we know that when the external inputting negative entropy equals to internal increasing entropy within a certain time period,that is, 


$$
\frac{\Delta Q_{e}}{\Delta t}=\frac{\Delta Q_{i}}{\Delta t}
$$

In this case,the internal increasing entropy is offset by the external inputting negative entropy,thus,

$$
\Delta s_{e}=\Delta s_{i}, \Delta s=0
$$

The enterprise system keep relative stability.The enterprise would keep scilent in fulfilling social responsibility,remain impervious to the government's propaganda and guidance and public opinion's supervision. So the enterprise may do nothing.

When the external inputting negative entropy is less than internal increasing entropy within a certain time period,that is,

$$
\frac{\Delta Q_{e}}{\Delta t}<\frac{\Delta Q_{i}}{\Delta t}
$$

In this case,some enterprises would like to pursue profit maximization and focus on cost saving and income improvement.The enterprise adjust by itself to reduce the investment in fulfilling social responsibility,let $\Delta s_{e}=\Delta s_{i}$, make the enterprise system reach negative equilibrium.

If some enterprises put profit above conscience,disregard of law and human security of life and properity, $\Delta s_{i}$ is greater than the absolute value of $\Delta s_{e}$, these enterprise would not like to maintain negative equilibrium,let $\Delta s>0$, the external inputting negative entropy can not offset the internal increasing entropy.Thus, the dissipative structure of the enterprise system is destroyed and the enterprise system is out of order.In this case,the enterprise will tread on social responsibility at any cost and do lots harm to the society and people.

When the external inputting negative entropy is greater than internal increasing entropy within a certain time period,that is,

$$
\frac{\Delta Q_{e}}{\Delta t}>\frac{\Delta Q_{i}}{\Delta t}, \Delta s_{e}>\Delta s_{i}, \Delta s<0
$$

Thus,the enterprise system form new dissipative structure,change from disorder to order and reach positive equilibrium.In this case,the enterprise adapt the trend of the times,fulfill economic,law,ethics and charity responsibility actively.So the enterprise build good social image,strengthen competitive power and achieve sustainable development [8, 9].

\section{The Coordination in the System When Fulfilling Social Responsibility}

Assuming the manufacturer face the consumer market directly, the risk of the supplier and the manufacturer is neutral,the supplier have full supply availability.The sale volume depends on selling price and the effort cost which the supplier and the manufacture fulfill social responsibility.The supplier makes to order which comes from the manufacturer and the purchased spare part which provide by the supplier is the key part for the production of the manufacture[10].

The purpose of the supplier and the manufacturer undertake social responsibility is to improve their brand image,corporate value and increase market demand.So assuming demand function is $D=D\left(p, S_{s}, S_{m}\right), \quad p$ is the selling price of per unit product produced by the manufacturer, $S_{s}$ is the level which the supplier fulfill social responsibility, $S_{m}$ is the level which the manufacturer fulfill social responsibility[11].

The demand function $D=D\left(p, S_{s}, S_{m}\right)$ depends on the selling price of per unit product of the manufacturer $p$ and corporate social responsibility level $S_{s}$ and $S_{m}$, assuming it is:

$$
D=D\left(p, S_{s}, S_{m}\right)=g(p) \cdot S\left(S_{s}, S_{m}\right)
$$

$g(p)$ show the influence of the selling price of per unit product of the manufacturer on the market demand, $S\left(S_{s}, S_{m}\right)$ show the influence of corporate social responsibility level on market demand.The study considers the relationship between the price and corporate social responsibility 
and consumption is multiple.Further,assume that $g(p)$ is linear decrease on $p$,specially set it to be:

$$
g(p)=(1-B p)
$$

$B$ is positive constant.For the simple expression,let the maximum value of $g(p)$ equal to 1 ,in addition,in order to ensure $g(p)>0$, limit $p<\frac{1}{B} \cdot S\left(S_{s}, S_{m}\right)$ is the influence of social responsibility level on market demand,is a nondecreasing function of $S_{s}$ and $S_{m}$, and have characteristics of concave function on $S_{s}$ and $S_{m}$. The more the $S_{m}$, the more influence on market demand form the level which the manufacturer fulfill social responsibility. As such,the more the $S_{s}$, the more influence on market demand form the level which supplier fulfill social responsibility.Let:

$$
S\left(S_{s}, S_{m}\right)=\left(k_{s} \sqrt{S_{s}}+k_{m} \sqrt{S_{m}}\right)
$$

$k_{s}$ and $k_{m}$ are positive constants,reflect the influence efficiency of the level of corporate social responsibility to market demand.

Combine (1)-(3),thus,

$$
D=D\left(p, S_{s}, S_{m}\right)=g(p) \cdot S\left(S_{s}, S_{m}\right)=(1-B p)\left(k_{s} \sqrt{S_{s}}+k_{m} \sqrt{S_{m}}\right)
$$

Assuming the unit production cost of spare part is $c_{0}$, the unit production cost of manufacturer is $c_{1}$, the price of supplier's spare part ordered by the manufacturer is $\omega$,the market price of unit product is $p$. The manufacturer encourage the supplier to fulfill social responsibility,and give certain subsidies.The cost proportion which the manufacturer share caused by the supplier fulfill social responsibility is $t(0 \leq t \leq 1)$. Without loss of generality,let $p>\omega>c_{0}$.

Based on above assumption and describtion, the single-period profit function of the manufacturer, the supplier and the whole supply chain system are respectively:

$$
\begin{aligned}
& D=\Pi_{s}=\left(\omega-c_{0}\right)(1-B p) \cdot\left(k_{s} \sqrt{S_{s}}+k_{m} \sqrt{S_{m}}\right)-(1-t) S_{s} \\
& \Pi_{m}=\left(p-c_{1}-\omega\right)(1-B p) \cdot\left(k_{s} \sqrt{S_{s}}+k_{m} \sqrt{S_{m}}\right)-t S_{s}-S_{m} \\
& \Pi=\Pi_{s}+\Pi_{m}=\left(p-c_{1}-c_{0}\right)(1-B p) \cdot\left(k_{s} \sqrt{S_{s}}+k_{m} \sqrt{S_{m}}\right)-S_{s}-S_{m}
\end{aligned}
$$

When the supplier and the manufacturer have the same decision-making power,they maximize their profit non-cooperatively at the same time. For the supplier,its single-period profit maximum model is:

$$
\begin{aligned}
& \operatorname{Ma}_{\omega, S_{s}} \Pi_{s}=\left(\omega-c_{0}\right)(1-B p) \cdot\left(k_{s} \sqrt{S_{s}}+k_{m} \sqrt{S_{m}}\right)-(1-t) S_{s} \\
& \text { s.t.p }<\frac{1}{B}
\end{aligned}
$$

For the manufacturer,its single-period profit maximum model is:

$$
\begin{aligned}
& \operatorname{MaX}_{p, S_{m}, t} \Pi_{m}=\left(p-c_{1}-\omega\right)(1-B p) \cdot\left(k_{s} \sqrt{S_{s}}+k_{m} \sqrt{S_{m}}\right)-t S_{s}-S_{m} \\
& \text { s.t. } 0 \leq t \leq 1, p<\frac{1}{B}, \quad p>c_{1}+\omega
\end{aligned}
$$

As we know from (9),the optimization solution of equilibrium satisfy the condition $t=0$.Clearly, $p \neq \frac{1}{B}$,if not, $\Pi_{s}=\Pi_{m}=0$.For solving above problem,the study use the conclusion drawn by Jorgensen and Zaccour(1999), that is,the supplier and the manufacturer have the same marginal cost 
when making decision at the same time.So:

$$
\left(p-c_{1}-\omega\right)=\left(\omega-c_{0}\right), \omega=\frac{p-c_{1}+c_{0}}{2}
$$

The first-order condition of the supplier and the manufacturer are:

$$
\begin{aligned}
& \frac{\partial \Pi_{s}}{\partial S_{s}}=\left(\omega-c_{0}\right)(1-B p) \cdot \frac{k_{s}}{2 \sqrt{S_{s}}}-(1-t)=0 \\
& \frac{\partial \Pi_{m}}{\partial p}=1-B p-B\left(p-c_{1}-\omega\right)=0 \\
& \frac{\partial \Pi_{m}}{\partial S_{m}}=\left(p-c_{1}-\omega\right)(1-B p) \cdot \frac{k_{m}}{2 \sqrt{S_{m}}}-1=0
\end{aligned}
$$

Solving the equation (11),(12) and (13), and notice $t=0$, we can get the only solution of Nash equilibrium( $\mathrm{N}$ show the Nash equilibrium):

$$
\begin{aligned}
& p^{N}=\frac{2+B\left(c_{0}+c_{1}\right)}{3 B} \\
& \omega^{N}=\frac{1+B\left(2 c_{0}-c_{1}\right)}{3 B} \\
& t^{N}=0 \\
& S_{s}{ }^{N}=\left(\frac{\left[1-B\left(c_{0}+c_{1}\right)\right]^{2} k_{s}}{18 B}\right)^{2} \\
& S_{m}{ }^{N}=\left(\frac{\left[1-B\left(c_{0}+c_{1}\right)\right]^{2} k_{m}}{18 B}\right)^{2}, \Pi_{s}{ }^{N}=\left(\frac{\left[1-B\left(c_{0}+c_{1}\right)\right]^{2} k_{s}}{18 B}\right)^{2}+2\left(\frac{\left[1-B\left(c_{0}+c_{1}\right)\right]^{2} k_{m}}{18 B}\right)^{2}, \\
& \Pi_{s}{ }^{N}=\left(\frac{\left[1-B\left(c_{0}+c_{1}\right)\right]^{2} k_{s}}{18 B}\right)^{2}+2\left(\frac{\left[1-B\left(c_{0}+c_{1}\right)\right]^{2} k_{m}}{18 B}\right)^{2} \\
& \Pi_{m}{ }^{N}=\left(\frac{\left[1-B\left(c_{0}+c_{1}\right)\right]^{2} k_{m}}{18 B}\right)^{2}+2\left(\frac{\left[1-B\left(c_{0}+c_{1}\right)\right]^{2} k_{s}}{18 B}\right)^{2}
\end{aligned}
$$

\section{Conclusion}

Based on Dissipative structure theory,the paper point out the formation of dissipative structure in enterprise system can improve the shortcoming and passivity in fulfilling social responsibility.

In the system, when the manufacturer does not cooperate with the supplier,in any case,the manufacturer is unwilling to share the costs which come from the supplier's social responsibility activities.Driven by self-interest,both the manufacturer and the supplier will invest capital and energies in social responsibility activities.The investment of the supplier and the manufacturer is related to the efficiency which their social responsibility level influence on market demand.When social responsibility activities are sensitive to market demand, the supplier and the manufacturer would like to invest more time,energy and capital in undertaking social responsibility.Thus, the supplier and the manufacturer will get more profits.So it is helpful for the system to form a dissipative structure in fulfilling social responsibility.

The more intensive research which combine it with CSR based on establishment of systematic 
equation in mathematics method is my further direction in the future.

\section{Acknowledgement}

This research is supported by my academic adviser, professor Li Jian.Thanks for his responsibility and patience. The author would like to thank the other authors provided resources.

\section{References}

[1]Prigogine I,Alan P M, "The Chanllege of Complexity,Self-organization and Dissipative Structure,”University of Texas Press, pp. 5-12, 1982.

[2]Haken H, “Information and Self organization,”Sichuan Education press,pp.55-62, 1988.

[3]Lida $\mathrm{Xu}$, YingFan and Zengru Di, "Cincepts,methods and applications of self-organization theory,”Journal of university of Shanghai for science and technology, vol.33(2),2011,pp. 130-136.

[4]Chunfeng Gao,“The study for rural community development in self-organization theory,”China agricultural university press,2009,pp.60-70.

[5]Faisal, Mohd. Nishat, "Analysing the barriers to corporate social responsibility in supply chains: an interpretive structural modelling approach”, International Journal of Logistics-research and Applications,vol.13,No,3,2010,pp. 179-195.

[6]Meng Jiong,Tang Dewo and Ni Xiaobing,"Safety responsibility strategy of manufacturer-retailer supply chain alliance based on profit distributive”,Journal of industrial engineering /engineering management, vol.24,No.1,2010, pp. 124-127.

[7]Smith, N. Craig,2010,“Marketing's Consequences: Stakeholder Marketing and Supply Chain Corporate Social Responsibility Issues”, Business Ethics Quarterly,vol.20,No.4,pp 617-641.

[8]Tate, Wendy L.Ellram, Lisa M.Kirchoff, Jon F,2010, “Corporate social responsibility reports: a thematic analysis related to supply chain management", Journal of Supply Chain Management,vol.46,No.1,pp 19-44.

[9]Lu Lan.,Yang Shuangyu, “Approach to the corporate social responsibility based on SCM,”Industrial engineering, vol.10, No.2, 2007,pp 31-37.

[10]Cruz Jose M, “The impact of corporate social responsibility in supply chain management: Multicriteria decision-making approach”, Decision Support Systems,vol.48,No.1,2009,pp. 224-236.

[11]Risso M, “A horizontal approach to implementing corporate social responsibility in international supply chains”, International Journal of Technology Management,vol.58, ,2012,pp. 64-82. 